\title{
Conflictos ambientales y respuestas sociales: el caso de reetnificación de la comunidad de Quillagua
}

\section{Bernardita Mc Phee Torres}

Diplomado en gestión socio-ambiental, complejidad y sustentabilidad, Universidad de Chile bernimcphee@gmail.com

\section{Resumen}

En el presente artículo se analiza la relación que existe entre el problema ambiental, ocasionado por la contaminación y presión que ejercen las mineras sobre la cuenca del curso medio del río Loa, y el surgimiento de la comunidad indígena de Quillagua, hecho que posibilita a su vez la aparición de un conflicto ambiental, a partir de las posibilidades legales y el respaldo internacional que brinda el hecho de ser indígena en contextos de conflictos ambientales. Asimismo, se propone que la etnicidad surge en los actuales escenarios como una herramienta de lucha y que por lo tanto los procesos de reetnificación o de etnificación se enmarcan dentro de dinámicas estratégicas e instrumentales.

\section{Abstract}

This article analyzes the relationship between environmental problem caused by pollution and pressure of mining companies in the basin of the middle of the river Loa, and the emergence of the indigenous community of Quillagua. This situation shows the emergence of an environmental conflict, reinforced by the legal possibilities and international backing provided by the fact of being indigenous. It is also proposed that ethnicity emerges in the current society as a tool of control and therefore the processes of ethnification or re-ethnification are part of an strategic and instrumental dynamic.

Palabras Clave: conflictos ambientales, sociedad, comunidades indígenas, etnificación, Quillagua Keywords: Environmental conflicts, society, indigenous communities, ethnification, Quillagua

\section{Introducción}

A partir de la década de los '90 en Chile, y en América latina en general, hemos observado el surgimiento de grupos ecologistas y activistas ambientales, que han ido introduciendo, en algunos sectores, el tema del medioambiente como un elemento central dentro de las movilizaciones sociales. De este modo, actualmente observamos que distintos movimientos sociales incorporan el discurso ambientalista dentro de sus movilizaciones, con lo que el "ecologismo" y la defensa del medioambiente han dejado de ser una demanda exclusiva de grupos ambientalistas para posicionarse como una necesidad y bandera de lucha de diversos sectores.

De este modo, de acuerdo a un estudio realizado sobre los conflictos sociales en Chile, analizados a partir de los medios de comunicación, los conflictos ambientales (tratados aquí como un tipo de conflicto cultural) son los que más han sido mencionados en 
distintos periódicos. Esta situación reflejaría que están presentes de manera importante en las comunicaciones de la sociedad, en la medida que reflejarían la opinión pública. (GÓMEZ, 2008)

De acuerdo a diferentes autores (Padilla y San Martín, 1994; Claude, 1997) el surgimiento de los conflictos ambientales, o de otra índole pero que utilizan la retórica ambientalista, se enmarca dentro del contexto de implantación de la economía neoliberal en los distintos países de Latinoamérica, que en el caso chileno ocurre en un escenario de dictadura y postdictadura. Esta situación se debería a que con la implantación de este modelo económico se ha presionado de manera importante los recursos naturales y con ello desencadenado importantes procesos de contaminación y/o degradación ambiental, ante los cuales han surgido respuestas y movilizaciones ciudadanas con el propósito de defender el medio ambiente.

Respecto a esto último existen importantes discrepancias teóricas, puesto que algunos plantean que efectivamente lo que busca defender la ciudadanía a través de la visibilización de conflictos y de las movilizaciones es el medioambiente, mientras que para otros lo que se pone en juego en los conflictos ambientales o socioambientales es mucho más que la conservación ecológica.

Dentro de los primeros se pueden destacar los planteamientos de Joan Martínez Alier y Ramachandra Guha a partir de la expresión "ecologismo de los pobres", que advierte sobre la defensa del medioambiente que hacen los pobres cuando ven amenazados los recursos naturales presentes en sus territorios. Dentro de los segundos se puede mencionar a Mauricio Folchi, quien plantea que los llamados conflictos ambientales en realidad responden a un conflicto de intereses en un medioambiente determinado (FOLCHI, 2001).

Pareciera evidente que en el contexto chileno los conflictos ambientales contienen diversos elementos y que lo que se pone en juego en "la lucha por el medioambiente" va mucho más allá de la conservación ecológica e incluso más allá del conflicto por el acceso y uso de los recursos naturales. De este modo, este tipo de conflictos nos enfrenta a discusiones que tienen que ver, entre otras cosas, con la distribución de la riqueza, con la inequidad social manifiesta en la participación desigual que tienen los diferentes actores sociales en la riqueza generada por los megaproyectos mineros, hidroeléctricos u otros; con los niveles de carencia que se observan en algunos sectores del país, etc. (SABATINI Y SEPÚLVEDA, 1997)

Al respecto es importante señalar, que en los conflictos ambientales locales existen múltiples niveles funcionando, por una parte necesidades y realidades locales, las que ya parece ambicioso unificar en un solo nivel, por otra, valores que la sociedad civil introduce con fuerza a nivel global como la conservación ambiental, la profundización de la democracia y la integración social; y además los intereses económicos de las empresas nacionales o corporaciones transnacionales y las regulaciones que imponen los Estados. Esta situación sin dudas hace que estos conflictos sean muy complejos y por ello muchas veces difíciles de resolver o de abordar. (SABATINI y SEPÚLVEDA, 2002)

Por otro lado, en Chile el rol del Estado frente a estos conflictos es limitado y en general hemos observado que el sector privado, por cumplir con las regulaciones ambientales impuestas por el Estado y/o por organismos internacionales, es quien negocia y procura mitigar o prevenir los impactos que distintos proyectos puedan causar, o bien, cooptar a los grupos afectados para evitarse conflictos ambientales. 
Esta situación ha llevado a que la ciudadanía tenga un papel central en estos conflictos y en general en "materia medioambiental" en la medida que son los propios actores sociales los que se vinculan con las empresas y buscan acuerdos de mitigación o compensación de los impactos asociados a los proyectos de inversión (Véase "Globalización, institucionalidad, estrategias, resistencia y conflictos ambientales", OLCA, 1999).

Desde un punto de vista político, el rol de los Estados en América Latina, en asuntos relacionados al medioambiente no es simple, puesto que en sociedades con importantes índices de pobreza es complejo tomar la decisión de detener el desarrollo económico en pos del medioambiente.

En el caso específico de Chile durante los últimos 10 años, hemos observado un crecimiento económico promedio del $6 \%$, situación que sin duda ha puesto en evidencia un fuerte proceso de sobreexplotación de los recursos naturales, ya que la economía nacional se basa en las exportaciones primarias intensivas en recursos naturales, lo que se verifica en cuatro sectores productivos primarios: minero, forestal, agrícola o pesquero. (QUIROGA, 2001).

Esta situación además se manifiesta a través de las movilizaciones de múltiples organizaciones o movimientos sociales que reclaman por el deterioro y por los daños ambientales que observan en sus territorios locales.

Pese a esta oposición, se observa que los proyectos de inversión de empresas nacionales o transnacionales, que son los que principalmente generan conflictos socioambientales, finalmente se realizan por más oposición que hayan logrado tener de parte de distintas organizaciones, lo que se observa en el dato de que de un total de 471 proyectos, entre los años 1997 y 2006, sólo un 7\% ha sido rechazado. (Rivera; 2009. en http://lasa.international.pitt.edu)

Esta situación genera una serie de dudas respecto a la capacidad de la institucionalidad chilena de frenar proyectos que no sean aceptables desde un punto de vista ambiental, y en general la discusión sobre el medioambiente en estos temas pasa por una crítica hacia la incapacidad del Estado de optar por el medioambiente en detrimento del crecimiento económico. Sin embargo hay un aspecto descuidado en estas ideas que tiene que ver con las respuestas sociales frente a estos conflictos, es decir, ¿qué papel tienen las comunidades locales en la resolución de estos conflictos.

Algunos autores señalan que las respuestas sociales o las movilizaciones de las comunidades que le dan origen a los conflictos ambientales tienen un sello más conservador que revolucionario. De este modo Sabatini señala que las movilizaciones ambientales: "...no proponen nuevos modelos de sociedad o estrategias de transformación social. Son, como se dijo antes, movimientos de reacción a los cambios profundos que está sufriendo la sociedad contemporánea, acicateados por el despliegue de la economía global. Representan la respuesta de la población al aumento masivo y polifacético de la inseguridad y la incertidumbre." (SABATINI, 1997: 11)

Al respecto Folchi señala que las respuestas sociales ante situaciones de conflicto ambiental no están atravesadas por la premisa de defender el medioambiente porque sea algo que se tiene que hacer por el beneficio del planeta, sino que las demandas tendrían que ver con lo que le conviene a los involucrados. En este sentido, de acuerdo al autor, en los conflictos de contenido ambiental operaría más una racionalidad económica que ambiental, es decir, los involucrados no asumirían una postura ética de 
proteger el medioambiente, sino que lo que se estaría reivindicando es el bienestar material objetivo de cada parte. (FOLCHI, 2001)

Sin embargo, es importante introducir en esta discusión que independiente de las conceptualizaciones teóricas que se establezcan, la sociedad interpreta estos conflictos como ambientales, y en este sentido es importante señalar que "lo ambiental" o bien lo que se entienda por medioambiente responde a construcciones sociales, por lo tanto dependerá del contexto histórico y cultural. De este modo Claudia Sepúlveda propone que: "... los conflictos ambientales se distinguen de otros conflictos sociales por el hecho de que sus contenidos sean interpretados como prioritariamente ambientales por el contexto histórico, social y cultural del que forman parte. En último término, el medio ambiente, así como las ideas que se tienen sobre él, son socialmente construidas, y por tanto, histórica y culturalmente acotables" (SEPÚLVEDA, 1997; 158)

\section{Ralco y Pascua Lama: algunos elementos para la discusión}

Un hecho interesante para la discusión sobre los conflictos ambientales es el conflicto ocurrido por la construcción y posterior operación de la central hidroeléctrica Ralco. Es interesante principalmente porque pareciera que a partir de esa experiencia las comunidades locales han sacado algunas cosas en limpio que les han permitido poner mayor oposición a los megaproyectos o bien emprender negociaciones exitosas con las empresas. En este sentido se podría señalar que Ralco sienta un precedente de lo que son las negociaciones económicas en la resolución de conflictos ambientales. Por otro lado el éxito de esta negociación estuvo estrechamente relacionado a la condición de indígena de los afectados, la que se constituyó en el recurso fundamental y en uno de los argumentos más potentes para mantener en el tiempo la oposición al proyecto. Por último, el hecho de que hayan sido comunidades indígenas las afectadas significó que estuvo presente en el conflicto una fuerte presión internacional exigiendo el respeto y la protección de los derechos indígenas. (Rivera; 2009. en http://lasa.international.pitt.edu)

En el caso de Pascua Lama, otro de los conflictos ambientales paradigmáticos en el país, al igual que en el conflicto de Ralco se observó que uno de los elementos más potentes para mantener la oposición al proyecto y encontrar acogida judicial internacional fue el hecho de que se estaba afectando a una comunidad indígena. Sin embargo a diferencia del caso de Ralco, lo llamativo de este conflicto es que por los mismos años que se inició el conflicto comenzó un proceso de reetnificación en la comunidad de los huascoaltinos, quienes mediante el recurso de la historia apelaron a una ocupación histórica del valle, con lo que el territorio se constituía como un territorio ancestral.

Es importante señalar, y uno de los temas que se busca discutir en este artículo es que el componente ambiental o discurso ambientalista dentro de las movilizaciones sociales muchas veces es utilizado como un mecanismo para movilizar viejas demandas sociales de diferente índole y que actualmente en algunos casos de conflictos ambientales se observa que las comunidades para tales fines desarrollan estrategias que les permiten posicionar "su conflicto" en la opinión pública y lograr un respaldo del sistema jurídico. Estas estrategias las hemos llamado respuestas sociales, las que no sólo son respuestas a un problema ambiental, sino que también son las que posibilitan que se pueda comunicar un conflicto, es decir, posibilitan que un grupo tenga la posibilidad de negar una expectativa (LUHMANN, 1998) 
Para realizar esta discusión, en el presente artículo se describe y analiza el caso de Quillagua, comunidad ubicada en el norte del país y que diariamente se enfrenta al problema de la contaminación y escasez de agua por la presión de ejerce la minería sobre este recurso.

Los datos utilizados para realizar este análisis fueron recopilados de fuentes secundarias y primarias. Entre las primeras se encuentra información del censo nacional de población y vivienda de 1992 y 2002, de la encuesta de caracterización socioeconómica nacional de 2006, revisión de medios de comunicación, entre las principales. La información primaria fue obtenida a través de entrevistas semiestructuradas aplicadas directamente en una campaña de terreno realizada en la localidad de Quillagua en enero de 2009.

A partir de los datos censales y del análisis intercensal se observó que hubo importantes cambios demográficos en la localidad, principalmente expresados en el decrecimiento poblacional y en el aumento de la población anciana en la localidad y disminución de niños y adultos. Por otro lado, se observa que entre los años 1992 y 2002 hay un importante aumento de personas que adscriben a un grupo étnico.

Sobre estos datos, en la campaña de terreno se indagó sobre las causas de los cambios sufridos en la localidad, con lo que se constató la existencia de un conflicto entre la comunidad y las diferentes empresas mineras de la zona. Este conflicto se comunica como un conflicto por el agua y desde retóricas ambientalistas.

Posteriormente se ha seguido este conflicto a través de los medios de comunicación, por una parte noticias que aparecen en periódicos regionales y por otra, distintas páginas web como páginas de ONG's y blogspot de jóvenes de Quillagua que emigraron de la localidad pero que asisten todos los años a la fiesta patronal y para las vacaciones de verano o de invierno.

\section{Antecedentes del conflicto ambiental de Quillagua}

La localidad de Quillagua administrativamente pertenece a la comuna de María Elena, Provincia de Tocopilla, Región de Antofagasta y se caracteriza por ser un valle, también denominado oasis, emplazado en el curso medio del río Loa, en plena zona desértica del Norte de Chile.

El asentamiento corresponde a una entidad poblada que históricamente ha estado ligada al trabajo agrícola, principalmente a la producción de maíz y alfalfa. Esta actividad se desarrollaba de manera intensiva, al punto de que hasta la década de los `60 Quillagua tuvo un rol fundamental en la región como abastecedora de productos agrícolas y como centro forrajero. Los sectores donde se desarrollaba la agricultura eran los predios de los comuneros, donde cada familia tenía su chacra. (PLADECO 2004 -2007)

Actualmente sólo el $10 \%$ de las tierras de cultivo está en manos de los habitantes de la localidad, puesto que el otro restante fue vendido por los pobladores a la empresa minera Soquimich. Esta situación significó además la venta de los derechos de agua, con lo que dicha empresa actualmente es propietaria de cerca del $80 \%$ de los derechos de agua correspondientes a la localidad. La venta de los predios y de los derechos de agua ocurrió principalmente a mediados de la década de los noventa. (PLADECO 2004 - 2007) 
La razón de esta situación fue que el agua del río Loa estaba contaminada, por lo que la agricultura empezó a desaparecer. En vista de esta situación, los pobladores consideraron que era una opción razonable vender sus derechos de agua, puesto que ya no podían desarrollar las actividades económicas de antaño, por lo que además, parte de la población, decidió emigrar de la localidad.

La agricultura desapareció producto de xantato, químico utilizado en la minería de cobre. Por otro lado, esta contaminación exterminó los camarones del río, los que otrora fueron una fuente de ingresos importante en la localidad. En cuanto a la ganadería, al no producirse alfalfa, la población dejó de tener animales.

De acuerdo al informe emitido por el Servicio Agrícola y Ganadero (SAG) el año 2000, el xantato identificado en las aguas del río Loa, sólo podía ser adjudicado a la actividad metalúrgica industrial, específicamente a la minería del cobre, lo que indicaría que el responsable de la contaminación fue Codelco, ya que en esos años era la única empresa de cobre cercana al río. Sin embargo Codelco no se ha responsabilizado por estos hechos, puesto que aparentemente aún no se ha podido determinar la responsabilidad de la empresa.

\begin{abstract}
"Ninguna autoridad se hizo responsable del desastre ambiental. La CONAMA regional identificó como causante del colapso ambiental al viejo tranque Sloman, un antiguo embalse abandonado y colmatado de sedimentos que sirvió de planta hidroeléctrica en épocas del salitre. Así le fue posible no investigar la responsabilidad de una institución intocable a nivel regional y nacional, pese a que las evidencias de los tipos de contaminantes apuntaron al tranque de relaves Talabre del mineral cobre de Chuquicamata, perteneciente a la Corporación Nacional del Cobre, CODELCO, lo que resulta evidente pues letales sustancias como el xantato y el isopropanol solo se utilizan en la industria del cobre." (www.portalchoapa.cl)
\end{abstract}

La crisis en Quillagua se observa al analizar y comparar algunos datos censales, los que muestran que, por una parte, ha disminuido la población y por otra que la población que existe actualmente son en su mayoría adultos mayores, por lo que los ingresos de la localidad corresponden en su mayoría a pensiones. De este modo, el año 2002, la población total de la localidad correspondía a 102 habitantes, observándose una variación negativa respecto al año 1992 de $-46,3 \%$. Así mismo, se observa una fuerte tendencia al envejecimiento de la población, lo que se explica por el éxodo masivo de población económicamente activa, posterior a la venta de los predios y de los derechos de agua. (PLADECO 2004 - 2007)

En este escenario crítico, se constituye el año 2003 la comunidad indígena aymara de Quillagua, con 25 socios, es decir, el mínimo que exige la ley indígena para que se constituya una comunidad. En el transcurso de los años ha aumentado significativamente el número de socios, siendo actualmente alrededor de 57 socios, lo que representa el $50 \%$ de la población de Quillagua. En relación a las cifras censales, se observa que en el año 1992 un 6,62\% de la población señaló pertenecer a la etnia aymara, mientras que en el censo de 2002 un 13,7\% declaraba adscribir a esta etnia, lo que refleja que entre los censos hubo una variación positiva de 7,11 puntos porcentuales. (Censo Nacional de Población y Vivienda 1992 y 2002)

La creciente identificación con la etnia aymara se explica en gran medida por la promulgación de la ley 19.253 o ley indígena de 1994. Sin embargo, a diferencia de otras comunidades indígenas en Chile, las que se constituyen principalmente a 
mediados de los noventa, se observa que la comunidad indígena aymara de Quillagua comienza un proceso tardío de constitución.

De acuerdo a la población esta situación se debe a que sus padres nunca les inculcaron la tradición indígena, puesto que no se autopercibían a sí mismos como "indios". Actualmente se observa que justamente es la población más joven la que está llevando a cabo el proceso de reconocimiento y recuperación de la tradición andina, mientras que la población adulta mayor no se reconoce a sí misma como aymara. De este modo, en algunas familias ocurre que los padres no están inscritos en la comunidad indígena y los hijos sí.

Al respecto, estudios antropológicos y arqueológicos han señalado que efectivamente la población de Quillagua no se reconoce a sí misma como indígena, sino que como "chilenos", y que aun cuando reconocen y valoran los sitios arqueológicos presentes en la localidad, especialmente los cementerios, no se sienten vinculados a cuyos muertos en términos de parentesco. Sin embargo existen antecedentes que indican que el origen de esta comunidad se encuentra en poblaciones indígenas vinculadas a la zona de Atacama y Tarapacá. (CARRASCO, AGÜERO, URIBE, AYALA y CASES, 2003)

En este sentido, se observa que en Quillagua se dio y se está dando un proceso fuerte de reetnificación, lo que les ha permitido acceder a una serie de beneficios del Estado y con ello, revertir, en cierto modo, la crisis socioeconómica.

\section{Procesos de reetnificación y conflictos ambientales}

Es importante tener en cuenta que, en términos generales, los procesos de etnificación y la etnicidad en sí son complejos y no responden en todos los casos a procesos de identificación basados en cuestiones esenciales, sino que por el contrario la etnicidad surge en los actuales escenarios latinoamericanos como una herramienta para la acción, es decir, como una estrategia de lucha contra la exclusión.

En el contexto latinoamericano los procesos de reetnificación están estrechamente relacionados a las dinámicas de la globalización, las que se han caracterizado, entre otras cosas, por la proliferación de organismos internacionales que han incorporado en sus discursos los derechos de los indígenas, propiciando un contexto internacional favorable a los derechos de estos. Respecto al tema medioambiental, muchos de estos organismos se sitúan en la ideología ecologista generada en la década de 1970 producto del revuelo mundial que generó el informe "Los límites del crecimiento", a partir del cual se han utilizado como modelo de referencia las formas de vida típicas de los pueblos originarios. (GÓMEZ, 2007)

En este sentido se puede plantear que los procesos de reetnificación en toda América Latina y las actuales movilizaciones indígenas se desarrollan cuando estos grupos encuentran un contexto político favorable y en la medida que el discurso ideológico que manejan para legitimarse y justificar sus derechos recibe fuerte aceptación entre algunos de los grupos de opinión dominantes en un determinado contexto. (CRISTOFFANINI, 2008)

De este modo se ha advertido que el "despertar de los grupos étnicos" o la "emergencia indígena" (BENGOA, 2000) debe entenderse no como una resurrección indígena, sino que como una manifestación de las crisis del sistema neoliberal en particular y de la modernidad en general. 
"Cuando hablamos de "despertar de los grupos indígenas" en México, no debemos pensar en una especie de resurrección de identidades indias por largo tiempo soterradas o en estado de hibernación, sino en la reinvención estratégica, por parte de dichos grupos, de una identidad colectiva en un contexto totalmente nuevo, como es el de un Estado neo-liberal que los excluye y margina en nombre de la modernidad" (GIMÉNEZ, 2000; 58)

En el caso concreto de la comunidad indígena de Quillagua, la condición de indígenas ha significado un elemento potente en el tema de los recursos naturales, en este caso el agua, ya que actualmente apelan, en contextos legales, a la propiedad ancestral de dicho recurso y del territorio, dos elementos que en cierta medida han podido proteger a partir de su condición legal de indígenas.

Esta misma situación, significa actualmente que todos los proyectos mineros de la zona y otros (por ejemplo un parque eólico), deban considerar en sus EIA la condición indígena de la población y por lo tanto someterse a la normativa vigente referida a grupos humanos protegidos.

En definitiva la condición de indígenas les ha permitido tener un mayor control sobre el territorio, ya que tanto a nivel nacional como internacional se reconoce que el territorio es un elemento central para el desarrollo de los grupos indígenas, y por lo mismo se han establecido una serie de exigencias respecto al respeto que debe existir hacia los territorios indígenas y también los recursos naturales presentes en ellos. (Véase Ley 19.253, Título II Del Reconocimiento, Protección y Desarrollo de las Tierras Indígenas y Artículo 64)

Si bien en términos jurídicos aún es débil la protección que reciben las tierras indígenas, de igual modo la Ley 19.253 o Ley Indígena es uno de los pocos instrumentos legales en Chile que permite proteger de alguna manera tierras una vez que han sido inscritas por una comunidad indígena en los Conservadores de Bienes Raíces. De este modo, la condición de indígena en Chile de una u otra manera les permite ejercer cierto control, tanto simbólico como material de los territorios que habitan e imponer una serie de restricciones, en este caso particular, a las mineras.

Por otro lado, además de la normativa nacional, es importante tener en cuenta la internacionalización de la cuestión indígena, lo que también ha aportado una batería de recursos legales para negar las expectativas de, en este caso, las mineras. Como veíamos brevemente en los casos de Ralco y Pascua Lama, este elemento fue uno de lo centrales para mantener la oposición a los proyectos hidroeléctricos y mineros.

Sobre este tema es interesante el planteamiento de Christian Gros, quien señala que:

"Si bien es cierto que la apertura económica contribuyó a acelerar la crisis de la pequeña producción campesina e indígena y que la presión de los conglomerados internacionales sobre los recursos naturales nunca fue tan fuerte (...) surgen también fuerzas nuevas y contrarias en el ámbito internacional, más favorables a la elaboración de una respuesta indígena" (GROS, 2000; 177)

Estos aspectos favorables a la elaboración de una respuesta indígena tendrían que ver con tres aspectos: i) existe una preocupación internacional sobre el medio ambiente, por lo tanto, por ejemplo, la presión que ejercen las mineras sobre los recursos hídricos no aparece como un problema que afecta exclusivamente a sus "dueños 
tradicionales", sino como un problema del planeta entero. ii) hay una fuerte preocupación por el reconocimiento y respecto de la diversidad cultural, donde aparece un nuevo actor expresado en la presencia de ONG especializadas en la defensa del medio ambiente, derechos humanos, promocionando formas alternativas de desarrollo, que en este contexto es denominado "etnodesarrollo". iii) el desarrollo de nuevos derechos positivos internacionales como el convenio 169 de la OIT o la declaración sobre derechos de los pueblos indígenas de la ONU. (GROS, 2000)

De acuerdo al autor, en este escenario marcado por la preocupación por la ecología, la biodiversidad y la diversidad cultural, los derechos humanos y el derecho a llevar procesos de desarrollo alternativos, la población indígena ocupa un lugar simbólico y estratégico creciente que le permite obtener nuevos recursos, ya sea económicos, organizativos, políticos y/o discursivos. Estos orientarían las movilizaciones indígenas, por lo que los Estados y los actores económicos se ven obligados a reorientar las políticas referidas a la cuestión indígena. (GROS, 2000). En definitiva se observa que existe un escenario internacional positivo en relación al tema de los derechos de los pueblos originarios y una imagen sobre ellos que los posiciona como los preservadores históricos del medioambiente de los recursos naturales. (CRISTOFFANINI, 2008)

En este sentido plantea que los efectos de la globalización son contradictorios, puesto que por una parte afecta a las comunidades indígenas, aumenta sus crisis y las obliga a reaccionar y a defenderse y por otro entrega herramientas y recursos que son aprovechados por ellas y sus organizaciones. En el caso de la comunidad de Quillagua esto se observa en que por una parte la contaminación y sobreexplotación de los recursos naturales de su territorio han provocado una crisis social importante, desestabilizando las dinámicas sociales de la comunidad entera. Sin embargo a la vez la globalización, les entrega actualmente una serie de herramientas para defenderse y defender su territorio, que como vimos, se relacionan a las posibilidades y protección que tienen los grupos indígenas principalmente a nivel internacional y en menor medida nacional.

Esta propuesta teórica, resulta muy sugerente al momento de analizar el proceso de reetnificación en Quillagua como respuesta a una crisis económica, social y ambiental, puesto que más allá de poder recibir los beneficios del Estado o de poder exigir compensaciones a las empresas, la adscripción a una etnia y consecuente constitución legal de la comunidad indígena, facilita la aparición de un conflicto, es decir, entrega las herramientas para que un grupo tenga la posibilidad de negar las expectativas del otro.

En este sentido es importante señalar que todo conflicto surge a partir de una contradicción que se comunica, de la negación de contenidos y expectativas sociales, por lo tanto un conflicto es un procedimiento de comunicación, refiere a un "no" comunicado. Sin embargo un elemento básico para que surja un conflicto es la posibilidad que exista dentro de un sistema social de comunicar el rechazo de una comunicación precedente, es decir, para que surja un conflicto un grupo debe tener la capacidad de comunicar un "no". (LUHMANN, 1998)

En este caso puntual la contradicción que se comunica es de parte de las comunidades locales, las que, mediante una serie de acciones, les comunican a las mineras que ya no podrán seguir contaminando y extrayendo agua, e interviniendo sus territorios. De esta forma se produce la negación de las expectativas de las mineras, las que se encuentran dentro del sistema económico y funcionan de acuerdo a las estructuras y programas de este. 
Sin embargo en el caso específico de la comunidad de Quillagua, la única posibilidad de establecer el rechazo y negar las expectativas de las mineras es mediante su constitución como comunidad indígena, puesto que actualmente en la legislación chilena e internacional están ampliamente protegidas, e incluso uno de los pocos, sino el único instrumento legal que protege un territorio (que por cierto no tiene que ver con el de propiedad privada) es la ley 19.253, que en el caso además de las comunidades indígenas del norte grande, vale decir, aymaras, atacameñas y quechuas, protege el "agua indígena", categoría excepcional, puesto que en la constitución el agua es un bien nacional y de uso público, por lo tanto de todos y de nadie.

Por supuesto que en la práctica esto último no en todos los casos significa una real protección hacia los recursos hídricos comprendidos en territorios indígenas, ya que mediante los derechos de aprovechamiento, el agua entra en el mercado y estos derechos sí constituyen propiedad, y si existen derechos de aprovechamiento sobre los recursos hídricos inscritos con anterioridad por terceros, estos no pueden ser reclamados por la comunidad. Sin embargo, funcione en todos los casos o no, la ley indígena es uno de los pocos instrumentos legales que al menos pretende proteger recursos naturales y territorios que pertenezcan a grupos indígenas y que los indígenas utilizan como recurso legal para defender los recursos naturales presentes en sus territorios.

Retomando la idea anterior, se observa que la contradicción, en el caso de la comunidad de Quillagua, para ser comunicada, primero se debe producir una evolución, que en este caso es el proceso de reetnificación de la comunidad. Posterior a esto, se puede comunicar la contradicción de una manera más eficiente en la medida que el sistema jurídico la respalda. De igual modo se observa que la tensión estaba presente antes de la constitución de la comunidad indígena, los comuneros observaron como se murieron sus plantaciones de alfalfa y maíz y evidenciaron que las piscinas se secaron, sin embargo muchos de ellos decidieron irse de la localidad en busca de nuevas posibilidades laborales y vendieron sus parcelas y sus derechos de agua, es decir, podríamos plantear que en la medida que no fue comunicada la contradicción no hubo un conflicto. El conflicto se materializó en la medida que la comunidad comunicó la contradicción, hecho que pudo ocurrir una vez que la comunidad se constituyó como comunidad indígena.

En este contexto la contradicción comunicada adquiere mayor fuerza en la medida que el sistema regulador de conflictos por excelencia en los sistemas sociales complejos es el sistema jurídico, y como dijimos este establece una protección a los indígenas del país. Al respecto, es importante recalcar que la contradicción se vuelve posible cuando un sistema dispone de la capacidad de negar.

La aparición de conflictos, considerando los procesos de limitación y regulación de estos, ha permitido que la sociedad evolucione hasta lograr la complejidad actual. Por lo tanto, la evolución requiere siempre una contradicción, es decir, que exista la posibilidad de negar expectativas sociales y producir así una variación evolutiva. En este sentido, evolución se entenderá como un cambio estructural de la sociedad, que surge a partir de variaciones seleccionadas y reestabilizadas, es decir, que se han formado en estructura. (LUHMANN y DE GEORGI, 1993)

En este caso, la evolución se manifiesta en el proceso de constitución de la comunidad indígena, lo aumenta la complejidad dentro del sistema social. En este sentido se 
entenderá que la evolución del sistema se relaciona directamente con las respuestas sociales frente a los conflictos, que en este caso particular toma la forma de iniciar un proceso de reetnificación con una finalidad concreta.

Sobre los procesos de evolución, la teoría de sistemas sociales propone tres mecanismos de la evolución: variación, selección y reestabilización. La variación hace referencia a los elementos del sistema social, es decir, a las comunicaciones. La selección se refiere al proceso de aceptación o negación de la variación, por lo tanto hace referencia a las estructuras del sistema. Por último, si la selección de la variación es positiva y logra establecer una formación estable, se habla de reestabilización. Es importante señalar que Luhmann plantea que si se produce una variación necesariamente se produce la evolución, para lo que es requisito fundamental que los procesos de variación y selección permanezcan separados (LUHMANN y DE GEORGI, 1993)

Por lo tanto, en el caso de la comunidad indígena de Quillagua, la variación, entendiendo esta como el proceso de conformación de la comunidad indígena, surge como respuesta a la irritación o perturbación proveniente del entorno, que en este caso son las empresas mineras que ejercen presión sobre el ecosistema de la localidad. Cabe destacar que las variaciones de las comunicaciones están íntimamente relacionadas a las negaciones, es decir, a través de las negaciones se rechazan proposiciones comunicacionales y con ello se posibilita la introducción de elementos nuevos o bien comunicaciones inesperadas.

Esta variación es aceptada mediante el proceso de selección, principalmente porque encuentra acogida en el sistema jurídico, generándose una formación estable. A partir de esta condición, la comunidad de Quillagua logra tener la posibilidad de negar las expectativas de las mineras y generar un conflicto.

\section{Transformaciones sociales en escenarios de conflictos ambientales: ¿avanzando hacia una "ciudadanía verde"?}

A partir de lo expuesto, vemos que actualmente no es extraño que se formen comunidades indígenas o como hemos señalado, que se den procesos de reetnificación en escenarios de conflictos ambientales, puesto que existe a nivel internacional una preocupación por la cuestión indígena y con ello, se han creado una serie de decretos, convenios, entre otros instrumentos que favorecen y estimulan la respuesta de las organizaciones locales, que en este caso es la constitución en tanto indígenas.

En este sentido se observa que la economía política del capitalismo moderno ha transformado y está transformando los espacios que podríamos denominar locales, lo que se observa diariamente en Chile a través de los conflictos en distintas localidades por las intervenciones de empresas nacionales y transnacionales. Sin embargo, debemos tener en cuenta que no se trata de que la economía determine todo. Los distintos grupos afectados por las imposiciones de los procesos político-económicos están reaccionando, y en este sentido, los territorios y los procesos de desarrollo local, en algunos casos, son coproducidos.

Es cierto que muchas veces la toma de decisiones de los grupos afectados se orienta hacia estrategias de sobrevivencia, en otros casos se inclinan hacia acciones políticas que intentan resistir y renegociar los procesos políticos y económicos impuestos. En los dos casos, esta capacidad de tomar ciertas decisiones y de negociar por parte de las 
organizaciones indígenas cambia los efectos de estos procesos sobre los territorios y el medio ambiente.

En contextos de comunidades indígenas se observa con mayor facilidad que los procesos de desarrollo ya no son completamente impuestos, en gran medida debido a que la legislación indígena chilena otorga a los indígenas una serie de derechos sobre sus territorios y recursos naturales, además de beneficios económicos que se expresan en becas para educación, vivienda, y una serie de proyectos concursables destinados al "desarrollo indígena", planes de desarrollo integral, entre otros. Por otro lado en los últimos meses se ha visto un gran revuelo por el comentado convenio 169 de la Organización Internacional del Trabajo, donde se reconocen derechos ancestrales a las comunidades indígenas, derechos sobre territorios, recursos naturales, derecho a la participación y a la consulta. Aunque falta mucho por hacer en materia de participación ciudadana, sin duda que estos avances han ayudado a que en algunos casos se incorporen las inquietudes y necesidades de las comunidades.

Por lo tanto existen y se están ampliando, los instrumentos legales que permiten a los indígenas controlar sus territorios, tomar ciertas decisiones respecto de hacia donde van dirigidas sus expectativas, las que, como hemos observado, se traducen fundamentalmente en profundizar los derechos sobre los territorios que han ocupado históricamente.

En el caso puntual de la comunidad de Quillagua se observa que se han desarrollado planes de reconversión económica, mejoramiento del acceso a servicios básicos, puesta en valor de sitios culturalmente significativos, entre otras cosas. Respecto al tema medioambiental se observa que los comuneros han establecido relaciones con ONG's, grupos ambientalistas, han participado en seminarios, etc. En definitiva, se observa que existe un fuerte activismo ecológico en algunos miembros de la comunidad. Sin embargo es posible apreciar, que estas acciones ecológicas se orientan principalmente y tienen la finalidad de defender y controlar el territorio, más que a defender el medioambiente propiamente tal. En este sentido es posible plantear que el discurso ambientalista dentro de las movilizaciones sociales es utilizado principalmente para articular otro tipo de demandas.

Es cierto que un punto muy importante de la discusión es que los intereses y valores de los principales actores involucrados se puedan expresar igualitariamente e incidir en la toma de decisiones, y que hay que seguir avanzando en materia de participación ciudadana y descentralización en la toma de decisiones, sin embargo es fundamental que exista una conciencia ambiental en la población para que las decisiones tengan que ver con la búsqueda del desarrollo sustentable y no sólo con negociaciones económicas muchas veces corto plazistas.

Por lo tanto, teniendo en cuenta las posibilidades que existen actualmente para las comunidades indígenas en el diseño del desarrollo local en sus territorios, es importante avanzar en la conformación de una ciudadanía ambiental, es decir, que la población esté educada ambientalmente hablando. Esto es fundamental para avanzar en la consecución del desarrollo sustentable, puesto que si no existe una conciencia ambiental o racionalidad ambiental en la ciudadanía, en términos de Enrique Leff, los procesos de desarrollo local seguirán basándose en la racionalidad económica, lo que en términos concretos se traduce la mayoría de las veces en medidas asistencialistas que generan dependencia de las comunidades locales hacia las grandes empresas que instalan proyectos en las cercanías de sus localidades, acrecentándose por una parte los problemas ambientales y por otra no resolviéndose problemas sociales. RM 


\section{Bibliografía}

ARNOLD, MARCELO (Julio de 2009). Las organizaciones desde la teoría de los sistemas sociopoiéticos. Revista Cinta de Moebio, versión on-line ISSN 0717-554X. www.moebio.uchile.cl/32/arnold.html.

BARTOLOMÉ, MIGUEL (octubre de 2008). Los Pobladores del desierto. Genocidio, etnocidio y etnogénesis en la Argentina. En Revista Amérique Latine Histoire et Mémoire. № 10. versión en línea publicada el 2005. http://alhim.revues.org.

CARRASCO, CARLOS; AGÜERO, CAROLINA; AYALA, PATRICIA; URIBE, MAURICIO y CASES, BÁRBARA. 2003. Investigaciones en Quillagua: Difusión del conocimiento arqueológico y protección del patrimonio cultural. Revista Chungará (Arica) Volumen 35, No 2. pp. 321-326.

CRISTOFFANINI, PABLO. 2008. "Globalización y Etnicidad en América latina: El caso Boliviano", Revista Diálogos Latinoamericanos, Universidad de Aarhus. No 13. pp. 1 17.

FOLCHI, MAURICIO. 2001. "Conflictos de contenido ambiental y ecologismo de los pobres: no siempre pobres, ni siempre ecologistas", revista Ecología Política No 22, pp. $79-100$

GIMÉNEZ, GILBERTO. 2000. "Identidades étnicas estado de la cuestión"; en Los retos de la etnicidad en los estados-nación del siglo XXI. Ed. CIESAS, INI, M.A. México.

GÓMEZ, ÁGUEDA. 2007. "El discurso político indígena en América Latina", en Revista Desacatos No 24, Centro de Investigaciones y Estudios Superiores en Antropología Social, México, pp. $215-228$.

GÓMEZ, ANDRÉS. 2008. Sobre el carácter cultural de la emergencia de conflictos sociales en Chile. Revista MAD N 18, pp. 20 - 37.

GROS, CHRISTIAN. 2000. "Ser diferente para ser moderno o las paradojas de la identidad" en Los retos de la etnicidad en los estados-nación del siglo XXI. Ed. CIESAS, INI, M.A. pp. 171 - 195. México.

LUHMANN, NIKLAS. 1998. Los sistemas sociales: Lineamientos para una teoría general. Ed. Anthropos, España.

Observatorio Latinoamericano de Conflictos Ambientales, 1999. Globalización, institucionalidad, estrategias, resistencia y conflictos ambientales. Ponencia preparada para la Conferencia Electrónica FAO-FTPP-Comunidec: Conflictos Socioambientales: desafíos y propuestas para la gestión en América Latina". Quito, Ecuador. En www.rlc.fao.org

PADILLA, CÉSAR y SAN MARTÍN, CARLOS. 1994. "Conflictos Ambientales. Una oportunidad para la democracia" Observatorio Latinoamericano de Conflictos Ambientales. Santiago. 
CLAUDE, MARCEL. 1997. "Una vez más la miseria ¿Es Chile un país sustentable?" LOM, Santiago de Chile.

QUIROGA, RAYEN. 2000. La sustentabilidad socioambiental de la emergente economía chilena entre 1974 y 1999. Evidencias y desafíos. En Emir Sader (editor) El ajuste estructural en América Latina. Costos sociales y alternativas. Ediciones CLACSO, pp. 255 - 274. Buenos Aires.

RIVERA, CLAUDIO "La otra inequidad? Medio ambiente, desarrollo y conflictos ambientales en el Chile postransición" Universidad de los Lagos, Chile. Prepared for delivery at the 2009 Congress of the Latin American Studies Association, Río de Janeiro, Brasil, Junio de 2009. En http://lasa.international.pitt.edu

SABATINI, FRANCISCO Y SEPÚLVEDA, CLAUDIA. 1997. Conflictos ambientales entre la globalización y la sociedad civil. Publicaciones CIPMA, Santiago.

SABATINI, FRANCISCO. 1997. Espiral histórica de conflictos ambientales. En Conflictos ambientales entre la globalización y la sociedad civil. Publicaciones CIPMA, pp. 23 - 36. Santiago.

SABATINI, FRANCISCO y SEPULVEDA CLAUDIA. 2002. Conflictos Ambientales, entre la globalización y la sociedad civil. Publicaciones CIPMA, pp. 50 - 54. Santiago.

SEPÚLVEDA, CLAUDIA. 1997. Cultura y conflictos ambientales: la formación social de la demanda ambiental en el caso de Golden Spring. En Conflictos ambientales entre la globalización y la sociedad civil. Publicaciones CIPMA, pp. 157 - 180. Santiago.

Ley 19.253 Sobre Protección, Desarrollo y Fomento de los Indígenas.

Censo Nacional de Población y Vivienda de 1992 y 2002. Instituto Nacional de Estadísticas.

www.portalchoapa.cl 